\title{
Two-Dimensional Electron-Hole Liquid in Single Si Quantum Wells with Large Electronic and Dielectric Confinement
}

\author{
N. Pauc, ${ }^{1, *}$ V. Calvo, ${ }^{1, \dagger}$ J. Eymery, ${ }^{1}$ F. Fournel, ${ }^{2}$ and N. Magnea ${ }^{1}$ \\ ${ }^{1}$ DRFMC/SP2M, CEA-Grenoble, 17 Avenue des Martyrs, 38054 Grenoble Cedex 9, France \\ ${ }^{2}$ LETI/DTS, CEA-Grenoble, 17 Avenue des Martyrs, 38054 Grenoble Cedex 9, France
}

(Received 7 January 2004; published 8 June 2004)

\begin{abstract}
We report a luminescence study of the electronic properties of the 2D electron-hole liquid in crystalline $\mathrm{Si}$ quantum wells with $\mathrm{SiO}_{2}$ dielectric barriers. The Fermi-Dirac condensation of $e-h$ pairs into a metallic liquid is strongly enhanced by spatial localization. We present experimental evidence for the formation of liquid nanodroplets, with size increasing with $e$ - $h$ pair density. The quantum confined regime is observed for well width below $15 \mathrm{~nm}$. The data are analyzed in a confinement model that takes account of the band-gap renormalization by $2 \mathrm{D}$ many-body effects and the increase of the Coulomb interactions due to the dielectric mismatch between the $\mathrm{Si}$ well and the $\mathrm{SiO}_{2}$ barriers.
\end{abstract}

DOI: 10.1103/PhysRevLett.92.236802

In 1968, Keldysh [1] speculated that the equilibrium phase of $e$ - $h$ pairs might be a high density metallic liquid, and this acted as a catalyst to a very large number of theoretical and experimental studies of the properties of this new state [2]. The band structure of bulk Si and Ge provided all the ingredients needed to observe the FermiDirac condensation of electron-hole pairs into a quantum liquid: the long radiative lifetime (resulting from the indirect band gap) necessary to reach thermodynamic equilibrium and a high degeneracy and anisotropy due to the multiple valley structure of the conduction band helping to stabilize the metallic condensate at a fixed density and suppress excitonic pairing.

This original phenomenon in condensed matter physics was widely studied in bulk semiconductors [2] and still represents a unique system to test and compare manybody theories and experiments. However, until now, the lack of convenient Si based heterostructures with high potential barriers has been a difficulty for experimental studies of a dense $e-h$ plasma in a low dimensional system. The studies were then done essentially in direct band-gap III-V and II-VI quantum wells (QWs), where the short radiative lifetime (0.1-1 ns) limits the measurements to time resolved experiments with very high density of $e-h$ pairs [3].

In 1998, Tajima and Ibuka [4] showed by cw photoluminescence (PL) experiments that the $e-h$ liquid (EHL) phase can exist in $200 \mathrm{~nm}$ thick silicon on insulator (SOI) thin films. This artificial material, which was developed to improve the performance of complementary metaloxide semiconductor devices, is composed of a structurally perfect layer of crystalline silicon $(c-\mathrm{Si})$ with a typical thickness of $200 \mathrm{~nm}$ over a $400 \mathrm{~nm} \mathrm{SiO}{ }_{2}$ barrier transferred to an 8" Si wafer via molecular bonding [5]. By simply oxidizing the SOI film, one can obtain a crystalline Si well embedded in two dielectric barriers with a height of $3.2 \mathrm{eV}$ for the electrons and $5 \mathrm{eV}$ for the holes.

In view of the preliminary results of Tajima and given current abilities to manufacture high quality artifi-
PACS numbers: 73.21.Fg, 05.30.Fk

cial $\mathrm{Si} / \mathrm{SiO}_{2}$ heterostructures, there is a unique opportunity to reinvestigate experimentally the $e-h$ pair equilibrium states in spatially confined systems with an indirect band gap.

This Letter reports a low temperature PL study of $c$-Si QWs obtained by molecular bonding with $\mathrm{SiO}_{2}$ dielectric barriers and constitutes the first steps to understand the behavior of quasi-2D EHL. The original effect seen with this system is a clear distinction of the confinement effect in two regimes.

In the first regime called spatial confinement $\left(l_{z}>\right.$ $15 \mathrm{~nm}$ ), it is mainly the thermodynamics of the Fermi fluids that is predominantly affected by the reduced dimension. The ability to control the pressure of the carriers allows to reach the regime where there is no phase separation between free excitons (FE) and condensed $e-h$ pairs, contrary to ideal thermodynamic behavior in $3 \mathrm{D}$ where the size of the droplets is determined by the dynamical equilibrium between $e-h$ liquid and $\mathrm{FE}$ gas phases [2].

In the second regime called quantum confinement $\left(l_{z}<\right.$ $15 \mathrm{~nm}$ ), the quantum confinement effect on energy band edge positions is unambiguously demonstrated in these $c$-Si QWs structures with their amorphous/crystalline interfaces and dielectric barriers. The blueshift of the 2D-EHL recombination peak with decreasing $l_{z}$ is described within a model assuming a rigid shift of the band edge due to the quantization of the carrier motion but perturbed by the renormalization of the $2 \mathrm{D}$ band gap resulting from the exchange-correlation interactions [6]. We also consider the large dielectric mismatch between the $\mathrm{Si}$ well $\left(\epsilon_{\mathrm{Si}}=11.4\right)$ and the $\mathrm{SiO}_{2}$ barriers $\left(\epsilon_{\mathrm{SiO}_{2}}=\right.$ 3.9) which modifies the Coulomb interactions inside the plasma due to the polarization charges induced at the semiconductor-dielectric interfaces [7].

The (001) $\mathrm{Si} / \mathrm{SiO}_{2} \mathrm{QWs}$ are made by thermal oxidation of SOI wafers with a buried oxide of $400 \mathrm{~nm}$. The well thickness is defined by the "consumption" of the Si layer during oxidation, down to a value of $4 \mathrm{~nm}$ as measured by 
spectroscopic ellipsometry and $\mathrm{x}$-ray reflectivity with an accuracy of $0.5 \mathrm{~nm}$. The PL measurements are made between 1.8 and $30 \mathrm{~K}$ with the $351 \mathrm{~nm} \mathrm{UV}$ line of an $\mathrm{Ar}^{+}$laser with a power density varying from $0.5 \mathrm{~W} \mathrm{~cm}^{-2}$ to $7 \mathrm{~kW} \mathrm{~cm}^{-2}$. This particular excitation waveleng th provides strong absorption in the direct band gap of $\mathrm{Si}$, with a penetration length of $10 \mathrm{~nm}$ as deduced from the value of the absorption coefficient $\alpha=9 \times 10^{5} \mathrm{~cm}^{-1}$ at $\lambda=$ $351 \mathrm{~nm}$ [8]. This is important because it minimizes the PL signal from the substrate and fills the well homogeneously with photoexcited carriers because the diffusion length of excitons is several orders of magnitude larger than the well thickness. The presence of the underlying barrier and the small thickness of the QW greatly reduces the so-called "spatial problem" which gave rise to nonuniform distributions of $e-h$ drops, excitons, and free carriers in bulk material experiments. Surface recombination can be neglected due to the high structural quality of the thermally grown $\mathrm{Si} / \mathrm{SiO}_{2}$ interfaces and the fluctuations of the potential resulting from atomic roughness at the interfaces are considered only as possible seeds for the EHL condensation. Figure 1 shows the thickness dependence of the PL spectra of single $\mathrm{Si} / \mathrm{SiO}_{2} \mathrm{QWs}$

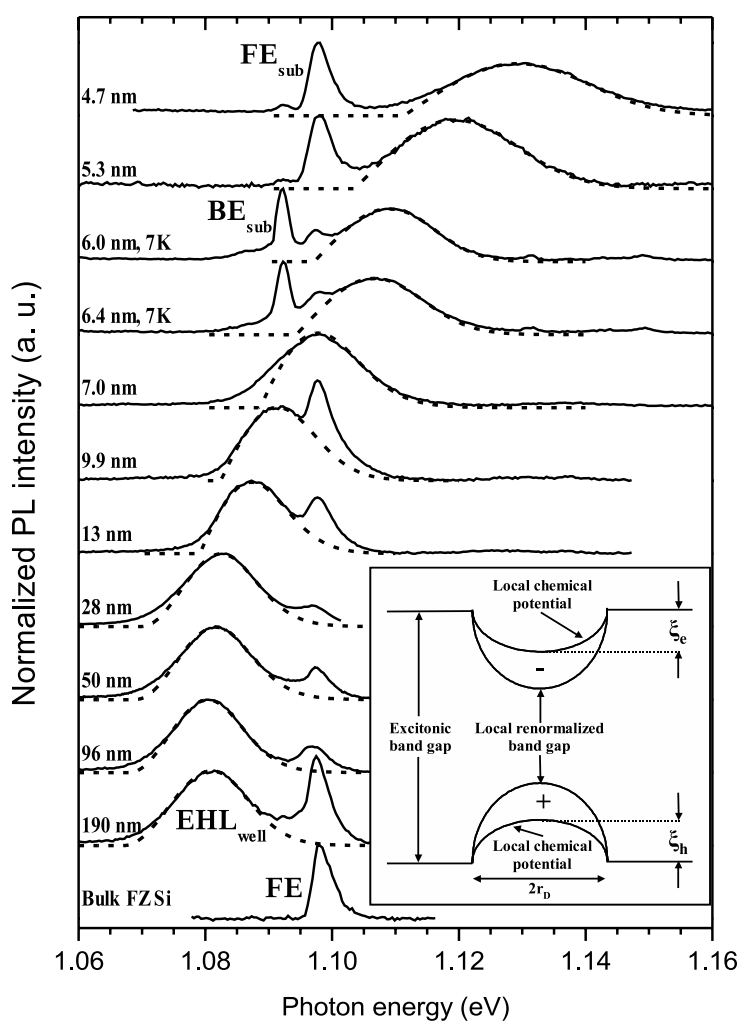

FIG. 1. PL of Si wells with $l_{z}$ varying between 4.7 and $190 \mathrm{~nm}$. Monitored temperature is $15 \mathrm{~K}$ (except other indication) and $P_{\text {ext }} \approx 7 \mathrm{~W} \mathrm{~cm}^{-2}$. The dotted curves are fits of the data following the 3D model for $l_{z}>13 \mathrm{~nm}$ and the 2D model for $l_{z} \leq 13 \mathrm{~nm}$. $\mathrm{FE}_{\mathrm{sub}}$ and $\mathrm{BE}_{\text {sub }}$ denote substrate free exciton and bound exciton recombination lines, respectively. Inset: representation of the "quantum droplet" of radius $r_{D}$ (case of parabolic potential for carriers). recorded in the energy region corresponding to radiative recombination with simultaneous $\mathrm{TO} / \mathrm{LO}$ phonon emission. The experiments are done principally at $15 \mathrm{~K}$ with a constant incident excitation density $P_{\mathrm{ext}}=7 \mathrm{~W} \mathrm{~cm}^{-2}$.

We first discuss the spatial confinement regime for $\infty>$ $l_{z}>3 a_{\mathrm{X}}^{\infty} \approx 15 \mathrm{~nm}\left(a_{\mathrm{X}}^{\infty}=4.9 \mathrm{~nm}\right.$ being the bulk exciton Bohr radius).

Here the ground state energy of the various phases of the $e-h$ pairs is the same as in an infinite 3D medium. By blocking the expansion of the photoexcited gas of carriers along $l_{z}$, the first effect of the spatial confinement is to favor the formation of the EHL as compared to bulk silicon which exhibits only a gas of noninteracting excitons free to expand in the volume of the sample. The EHL is identified by its distinctive slightly asymmetric band peaking at $1.082 \mathrm{eV}$, with the renormalized band gap $E_{B}^{*}-1 \hbar \omega_{\text {TO }}$ given by the low energy cutoff at $1.068 \mathrm{eV}$ [2]. From the spectroscopic data and the fit of the line shape (dotted line) which is determined to a good approximation by the density of occupied states of the distinct 3D Fermi liquids of electrons and holes, we obtain an $e$-h pair density $n=3.5 \times 10^{18} \mathrm{~cm}^{-3}$ and a binding energy of the liquid with respect to the exciton $\Phi=8 \mathrm{meV}$, in very good agreement with well established values measured and calculated in bulk Si [2].

In the spectra of Fig. 2(a) recorded for a $190 \mathrm{~nm}$ thick QW, the EHL line shape is independent of excitation level over a wide range of pumping power, due to the constant density of the liquid.
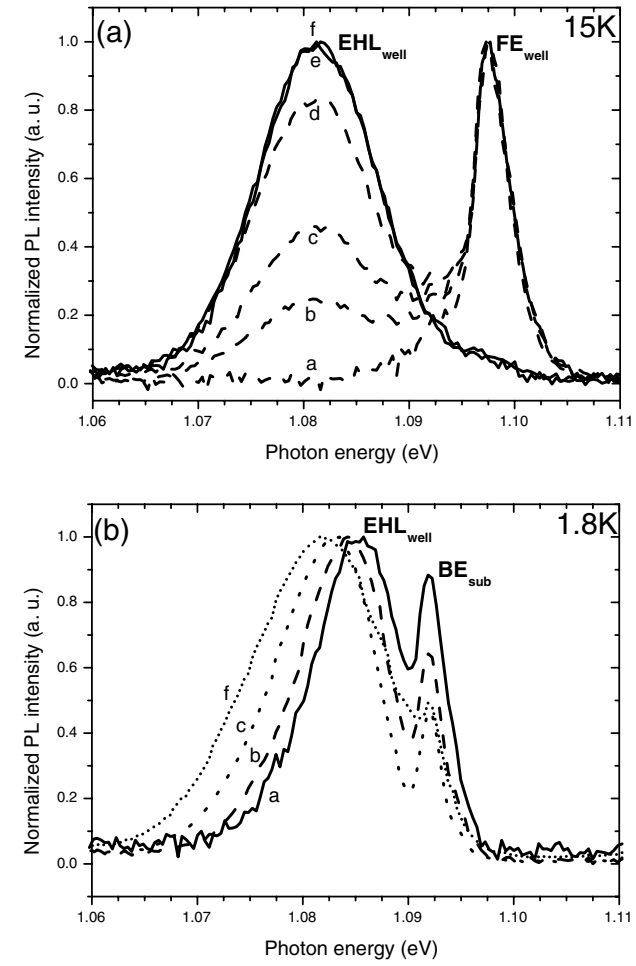

FIG. 2. PL of (a) a $190 \mathrm{~nm}$ well at $15 \mathrm{~K}$ and (b) a $28 \mathrm{~nm}$ well at $1.8 \mathrm{~K}$ for different laser power: $a=0.5, b=2, c=4, d=8$, $e=2.6 \times 10^{3}$, and $f=4 \times 10^{3}$, in $\mathrm{W} \mathrm{cm}^{-2}$. 
Three phases are clearly observed as a function of the $e-h$ pair density $N_{e h}=\eta(1-R) P_{\text {ext }} \tau /\left(l_{z} h \nu\right)$, with $\eta=1$ in the case of a thick QW $\left(\eta=1-\exp \left[-\alpha l_{z}\right]\right), R=0.55$ the reflection coefficient of the $\mathrm{SiO}_{2} / \mathrm{Si}$ dielectric stack, $\tau$ is either the EHL lifetime $\left(\tau_{\mathrm{EHL}}=0.5 \mu \mathrm{s}\right)$ or the FE lifetime $\left(\tau_{\mathrm{FE}}=2 \mu \mathrm{s}\right)$. We define a filling factor of the well by the liquid phase as the ratio of the $e$ - $h$ pair density to the equilibrium liquid density $F_{\text {liq }}=N_{e h} / n$.

For $N_{e h} \leqslant 2-3 \times 10^{15} \mathrm{~cm}^{-3}, F_{\text {liq }} \approx 10^{-3}$, we detect only the FE line in very good agreement with the phase diagram of the $e-h$ fluid in bulk Si [9]. This means that a single gaseous phase of noninteracting free excitons is stable in the well. Because the exciton density is too low and the lifetime $\tau_{\mathrm{EHL}}$ is finite, the recombination inside any liquid droplet cannot be compensated by the flow of $e-h$ pairs from the surface, and the droplet does not reach the critical Kelvin radius necessary to grow and reach a stable size.

For $10^{18} \mathrm{~cm}^{-3}>N_{e h}>4 \times 10^{15} \mathrm{~cm}^{-3}$, the liquid and the exciton gas phase coexist in the QW. The EHL peak increases steadily with $P_{\text {ext }}$ at the expense of the FE peak. For $N_{e h}>10^{18} \mathrm{~cm}^{-3}$, that is, a filling factor $F_{\text {liq }}$ close to 1 , the FE signal has disappeared and all the $e$ - $h$ pairs are condensed in the liquid phase which occupies a large part of the volume of the well. Similar behavior is observed when $N_{e h}$ is increased by decreasing the QW width while keeping the excitation power constant. As seen in Fig. 1 the EHL dominates the spectra while the FE emission coming from the QW disappears progressively when $l_{z}$ decreases. For $l_{z} \leq 30 \mathrm{~nm}$ the QW becomes partially transparent to the laser excitation, and we detect substrate emission at the $\mathrm{FE}_{\mathrm{TO} / \mathrm{LO}}$ or bound exciton $\left(\mathrm{BE}_{\mathrm{TO} / \mathrm{LO}}\right)$ recombination energy.

Contrary to the spectra of thicker wells where the position of the EHL peak is independent of excitation power, the spectra of Fig. 2(b), recorded at $1.8 \mathrm{~K}$ for the $28 \mathrm{~nm}$ QW, show a redshift of $5 \pm 1 \mathrm{meV}$ for the EHL as $F_{\text {liq }}$ increases from $10 \%$ to $100 \%$ [10]. We explain this phenomenon by the formation of nanodroplets of $e-h$ liquid with a size limited in one direction to the well width and increasing laterally with the filling factor.

In the standard model of large 3D EHL droplets, the size dependence of the energy per $e-h$ pair of a spherical EHL droplet of radius $r_{D}$ is given by the chemical potential $\mu\left(r_{D}\right)=\mu(\infty)+2 \sigma /\left(n r_{D}\right)$, where $\mu(\infty)$ is the chemical potential for an infinite drop of density $n$ and $\sigma$ is the surface energy density [2]. But in a QW at small $F_{\text {liq }}$, i.e., small $r_{D}, \mu\left(r_{D}\right)$ depends strongly on the droplet size. This explains qualitatively the redshift of the EHL peak towards a saturation value when the well is filled as a result of the coalescence of the droplets.

As it is difficult to define the surface energy $\sigma$ of nanometric droplets, a more appropriate description of this effect is to consider the $e$ - $h$ droplet as a quantum droplet where the carriers are confined by the band gap lowering inside the liquid relative to the outside coming from the effect of many-body interactions. In this model, the barrier heights are given by the work function $\xi_{e}$, $\xi_{h}$ of electrons and holes in the liquid, with $\xi_{e}=\xi_{h}=$ $\frac{1}{2} \Phi \approx 4 \mathrm{meV}$ in $\mathrm{Si}$ [2]. For simplicity, we represent the gradual potential which exists between the inside of the droplet and the surrounding medium (see the inset diagram in Fig. 1) by a parabolic profile. The ground state energy of a single particle immersed in the quantum droplet is simply the solution of the 3D harmonic oscillator $\delta E_{e, h}=\frac{3}{2} \hbar \omega_{e, h}=\frac{3}{2} \hbar \sqrt{8 \xi_{e, h} /\left(m_{e, h} l_{z}^{2}\right)}$, with $m_{e}=$ 1.08 and $m_{h}=0.55$ [2]. If we suppose that the $e$ - $h$ fluid does not wet the oxide, the smallest droplet is a sphere with diameter $2 r_{D}=l_{z}=28 \mathrm{~nm}$ limited by the wall, and the recombination line shifts to high energy by $\Delta E=$ $\delta E_{e}+\delta E_{h}=6.1 \mathrm{meV}$, compared to a laterally large droplet. Considering the simplicity of the model, the agreement with the experimental shift $(\Delta E=5 \mathrm{meV})$ supports our hypothesis about the formation of nanodroplets at low filling factor.

For the quantum confinement regime obtained for $l_{z}<$ $15 \mathrm{~nm}$, the ground state energy of an $e-h$ pair is now strongly modified. The $e-h$ recombination peak - which is now labeled EHP ( $e-h$ plasma) because of the undetermined nature of the $e-h$ phase, liquid or plasma - shifts to higher energy as seen in the spectra of Fig. 1, unlike the $\mathrm{FE}_{\text {sub }}$ one which is due for $l_{z}<28 \mathrm{~nm}$ to exciton recombination in the substrate. In Fig. 3 we have plotted (triangles) the variation of the maximum $E_{\max }$ of the 2D EHP peak. This maximum is linked to the band-gap renormalization (BGR) $E_{\mathrm{BGR}}$ by $E_{\max }\left(l_{z}\right)=E_{G}\left(l_{z}\right)-$ $E_{\mathrm{BGR}}\left(l_{z}\right)+\left(E_{F e}+E_{F h}\right) / 2-1 \hbar \omega_{\mathrm{TO}}$ [11], with $E_{F e}$ and $E_{F h}$ the Fermi levels of electrons and holes, respectively.

To our knowledge there is no full calculation of the confinement energy of a quasi-2D EHL in $\mathrm{Si} / \mathrm{SiO}_{2}$ quantum wells for comparison with our data. We use a model where the 2D Coulomb interactions are considered as perturbations to the quantum electronic confinement of single electron and hole states. The correction brought by

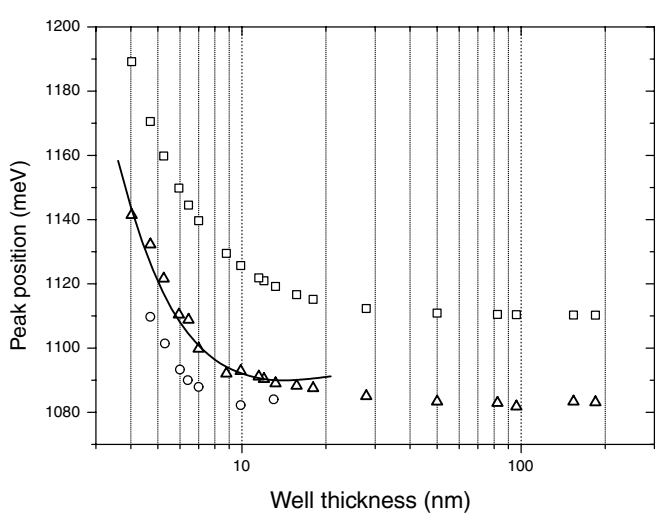

FIG. 3. Comparison between experimental and theoretical positions of the 2D EHP peak vs $l_{z}$. Squares: $E_{G}\left(l_{z}\right)-$ $1 \hbar \omega_{\mathrm{TO}}$; triangles: experimental $E_{\max }\left(l_{z}\right)$; circles and solid line: theoretical $E_{\max }\left(l_{z}\right)$ with and without dielectric mismatch respectively. 
the exchange and correlation contribution to the single particle self-energy depends strongly on the quasi-2D exciton binding energy $E_{\mathrm{X}}$ and the 2D Wigner-Seitz radius $r_{s}$ which both vary with the well width. First, the band gap $E_{G}\left(l_{z}\right)$ of the $\mathrm{Si}(001) \mathrm{QW}$ is taken from tight binding calculations [12]. The $l_{z}$ dependence of the EHL $0 \mathrm{~K}$ equilibrium density $n_{\mathrm{eq}}$ and the $2 \mathrm{D}$ band-gap renormalization $E_{\mathrm{BGR}}\left(l_{z}\right)$ are calculated within the model of Kleinman [6] based on an extension of the RPA theory [13] to the 2D case. We used $n_{\text {eq }}\left(l_{z}\right)$ to calculate the Fermi energies. These values are injected in the calculation of $E_{\max }\left(l_{z}\right)$ whose theoretical values are plotted in Fig. 3 (solid line) and are in a relatively good agreement with the experimental data for $l_{z} \leq 15 \mathrm{~nm}$. As an example, we get the following theoretical parameters of the $e$ - $h$ condensate for $l_{z}=4.7 \mathrm{~nm}, n_{\mathrm{eq}}=1.0 \times 10^{12} \mathrm{~cm}^{-2}, r_{s}=$ $1.14 a_{\mathrm{X}}^{\infty}$, and $\Phi=12.8 \mathrm{meV}$.

The experimental EHP density $n_{\exp }$ is obtained by numerical analysis of the line shape PL spectra using 2D Fermi levels for electrons and holes with a staircaselike density of states (see the dotted fitted curves in Fig. 1). By taking heavy hole masses $m_{h h}=0.21$ and electron masses $m_{e}=0.19$ [14] we obtain for the $4.7 \mathrm{~nm}$ well $n_{\exp }=2.4 \times 10^{12} \mathrm{~cm}^{-2}$ and $r_{s}=0.74 a_{\mathrm{X}}^{\infty}$ with $T_{f}=$ $38 \mathrm{~K}$ [15]. This density is noticeably higher than the calculated value $n_{\mathrm{eq}}=1.0 \times 10^{12} \mathrm{~cm}^{-2}$, indicating that $e-h$ interactions are imperfectly treated.

A more realistic model must account for the dielectric mismatch between the well and the barrier. In the particular case of $\mathrm{Si} / \mathrm{SiO}_{2} \mathrm{QW}$, the $e-h$ interactions should be enhanced not only by the carrier confinement but also by an increase of the Coulomb interaction caused by the image charges in the $\mathrm{SiO}_{2}$ barrier. The theory of Keldysh for excitons in semiconductor-dielectric nanostructures [7] is used to calculate $E_{\mathrm{X}}\left(l_{z}\right)$ and $a_{\mathrm{X}}\left(l_{z}\right)$ and to reevaluate the quasi-2D exchange-correlation energy of the EHL in the model of Kleinman. Values for $E_{\max }\left(l_{z}\right)$ taking into account the modification of the Coulomb interaction are shown in Fig. 3 (circles). They give a too strong renormalization of the gap, but the calculated equilibrium density $n_{\mathrm{eq}}=2.0 \times 10^{12} \mathrm{~cm}^{-2}$ is now close to that measured experimentally $n_{\exp }=2.1 \times 10^{12} \mathrm{~cm}^{-2}$. The two above approaches - unmodified and modified Coulomb interactions - suggest that the $e-h$ system takes the form of a 2D EHL whose density and band-gap renormalization have intermediate values between those given by the two models.

In conclusion, we have identified novel behavior of EHP under a 2D confinement. The transition from 3D to 2D not only modifies the free energy of the Fermi fluid but also the thermodynamic equilibrium between the different phases. A question that is still open is the nature of the phase in the quantum confined regime. Our calculation of the binding energy and the phase diagram of the EHL with the models of Kleinman [6] and Droz and
Combescot [16], respectively, predict that the metallic liquid phase is stable in $5 \mathrm{~nm}$ thick $\mathrm{Si} / \mathrm{SiO}_{2}$ quantum wells with a critical temperature of $60 \mathrm{~K}$. Experimental data are now needed to test this prediction. We point out that these $\mathrm{Si} / \mathrm{SiO}_{2}$ structures, if submitted to a longitudinal electric field, are particularly suitable to create two spatially separated layers of electrons and holes and thus to study the new condensed phases which could result from a coherent pairing of carriers [17]. Measurements of the $2 \mathrm{D}$ and $1 \mathrm{D}$ exchange-correlation interactions in the presence of electric and magnetic perturbations, nanofluidic properties of a spatially confined liquid of charged particles, and condensation of long-lived excitons in potential traps are among the problems which could now be treated by the experimentalist and compared with theoretical predictions.

\section{*Electronic address: pauc@drfmc.ceng.cea.fr}

†Electronic address: vincent.calvo@cea.fr

[1] L. Keldysh, in Proceedings of the 9th International Conference on the Physics of Semiconductors, Moscow, 1968 (Nauka, Leningrad, 1968), p. 1307.

[2] T. M. Rice, J. C. Hensel, T. G. Phillips, and G. A. Thomas, Solid State Physics (Academic Press, New York, 1977), Vol. 32.

[3] H. Kalt, K. Reimann, W.W. Rhüle, M. Rinker, and E. Bauser, Phys. Rev. B 42, 7058 (1990).

[4] M. Tajima and S. Ibuka, J. Appl. Phys. 84, 2224 (1998).

[5] M. Bruel, B. Aspar, and A. J. Auberton-Hervé, Jpn. J. Appl. Phys. 36, 1636 (1997).

[6] D. A. Kleinman, Phys. Rev. B 33, 2540 (1986).

[7] L. Keldysh, Phys. Status Solidi A 164, 3 (1997).

[8] D. E. Aspnes, Properties of Crystalline Silicon (Inspec, London, 1999).

[9] L. M. Smith and J. P. Wolfe, Phys. Rev. B 51, 7521 (1995).

[10] At $1.8 \mathrm{~K}$ the substrate emission corresponds to bound exciton recombination $\left(\mathrm{BE}_{\mathrm{sub}}\right)$, while at $15 \mathrm{~K}$ it corresponds to the free exciton $\left(\mathrm{FE}_{\mathrm{sub}}\right)$ line (see Fig. 1).

[11] We found numerically the energy difference between the maximum peak position of a 2D EHP and the renormalized band gap is half of the Fermi level of electrons and holes.

[12] Y. M. Niquet, C. Delerue, G. Allan, and M. Lannoo, Phys. Rev. B 62, 5109 (2000).

[13] W. F. Brinkman and T. M. Rice, Phys. Rev. B 7, 1508 (1973).

[14] T. Ando, A. B. Fowler, and F. Stern, Rev. Mod. Phys. 54, 437 (1982).

[15] We found a fit temperature $T_{f}=38 \mathrm{~K}$ - higher than the monitored temperature $15 \mathrm{~K}$-to account for the line broadening which may be due to sample heating by laser beam and $l_{z}$ variations of the excited zone leading to energy band-gap variations.

[16] M. Droz and M. Combescot, Phys. Lett. 51A, 473 (1975).

[17] Yu. E. Lozovik and V. I. Yudson, Sov. Phys. JETP 44, 389 (1976). 